\title{
A surgical approach to squamous cell carcinoma of penis that also resolved the psychological dysfunction of the patient
}

\author{
Napoleon Moulavasilis ${ }^{1}$, Konstantina Yiannopoulou ${ }^{2}$, Marios Frangoulis ${ }^{3}$, Ioannis Katafigiotis ${ }^{1}$, \\ Georgios Liapis ${ }^{4}$, Aikaterini Anastasiou ${ }^{5}$, Ioannis Anastasiou ${ }^{1}$ \\ ${ }^{1} 1^{\text {st }}$ Department of Urology, National and Kapodistrian University of Athens, Laiko Hospital, Athens, Greece; \\ 2 Department of Neurology, Henry Dunant Hospital Center, Athens, Greece; \\ ${ }^{3}$ Plastic Surgeon, Academic Fellowship, National and Kapodistrian University of Athens, Laiko Hospital, Athens, Greece; \\ ${ }^{4}$ Department of Pathology, Medical School of Athens, National and Kapodistrian University of Athens, Greece; \\ ${ }^{5}$ Medical School of Athens, National and Kapodistrian University of Athens, Greece.
}

\begin{abstract}
Summary In this case study, we present an unusual case with squamous cell carcinoma surrounding the penis involving foreskin and glans of penis. In addition, multiple satellite nodules were noted in the pubis. A 57-year-old circumcised heterosexual male patient presented with a penile lesion existing for 10 years. At the same time, he was referred to an outpatient memory clinic because of persistent subjective memory complaints associated with depression and anxiety. The patient was operated under general anaesthesia. The lesion was resected circumferentially with macroscopic clearance, resulting in complete degloving of the penile shaft. Neurovascular bundles were preserved. Histopathological analysis of the lesion revealed an invasive and poorly differentiated squamous cell carcinoma, and the surgical margins were free from tumour. The patient was followed for 18 months. He did not have voiding difficulty. Pelvic tomographic and physical examination findings did not reveal any episode of recurrence or metastasis. Treatment of carcinoma resulted in a simultaneous full recovery of his memory decline and he remained free of depression and anxiety symptoms over time.
\end{abstract}

KEY WORDS: Squamous cell carcinoma; Penis; Surgical technique.

Submitted 10 August 2019; Accepted 21 September 2019

\section{INTRODUCTION}

Penile carcinoma is an uncommon tumor which is devastating for the patient and therapeutically challenging for the urologist. Invasive penile carcinoma accounts for $0.4-0.6 \%$ of malignant neoplasms among men (1). Carcinoma of the penis usually begins with a small lesion that gradually extends to involve the entire glans, shaft and corpora. Patients with cancer of the penis, more than patients with other types of cancer, seem to delay seeking medical attention. Explanations include embarrassment, fear, guilt and ignorance (2). The oncologic gold standard for definitive treatment of the penile cancer is surgical amputation of the tumor (3).

Conservative surgery may be safely performed in well selected patients in order to maintain penile function and length. Additionally, penile cancer sufferers can exhibit significant preoperative and postoperative psy- chological dysfunction (4). However, it appears that by using established reconstructive surgical techniques we are able to achieve significant improvements of anxiety, depression (5) and subjective memory complaints.

\section{Case report}

A 57 years old heterosexual male patient presented with a penile lesion existing for 10 years. He has never asked for medical help. As the lesions became aggressive and eventually surrounded the penis, he referred to our department. He suffered from the huge mass, which prevented him from having sex and caused significant voiding diffculty. On physical examination, a tumoural mass resembling a cauliflower measuring nearly $8 \times 4 \mathrm{~cm}$ encircling the entire penile shaft and satellite nodules in pubis were detected (Figure 1). Clinically and on pelvic computed-tomography, no inguinal lymphadenopathy was noted. At the same time, he was obviously anxious and he also confessed that he suffered from persistent memory decline and depressive symptoms.

Consequently, he was referred to an outpatient memory clinic for complete preoperative neuropsychological evaluation. Neurological examination and Magnetic Resonance Imaging (MRI) of the brain were perfectly normal. Nevertheless, the neuropsychological questionnaires revealed a serious depressive and anxiety disorder. He scored 17/21 and 15/21 for anxiety and depression respectively in the Hospital Anxiety and Depression Scale (HADS) (6) and 11/15 the Geriatric Depression Scale (GDS) (7). On the contrary, questionnaires regarding memory difficulties and executive functions (7) were perfectly completed.

The patient was operated under general anaesthesia. A complete resection of the glands of penis was performed and the lesion was resected circumferentially with macroscopic clearance, resulting in complete degloving of the penile shaft. Neurovascular bundles were detected during the surgical procedure and preserved. The distal urethra sectioned by the circumcision was reconstructed, and the edges were everted, creating a vertical ure- 
Figure 1.

Penile lesion.

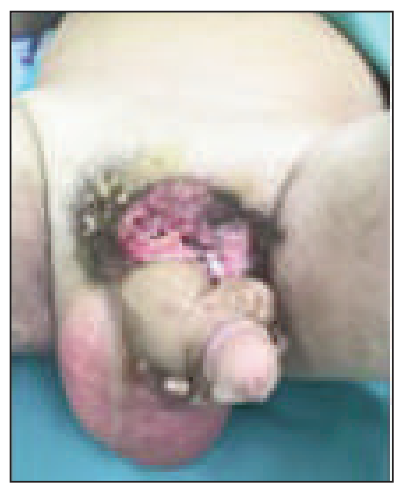

Figure 2.

Penile graft.
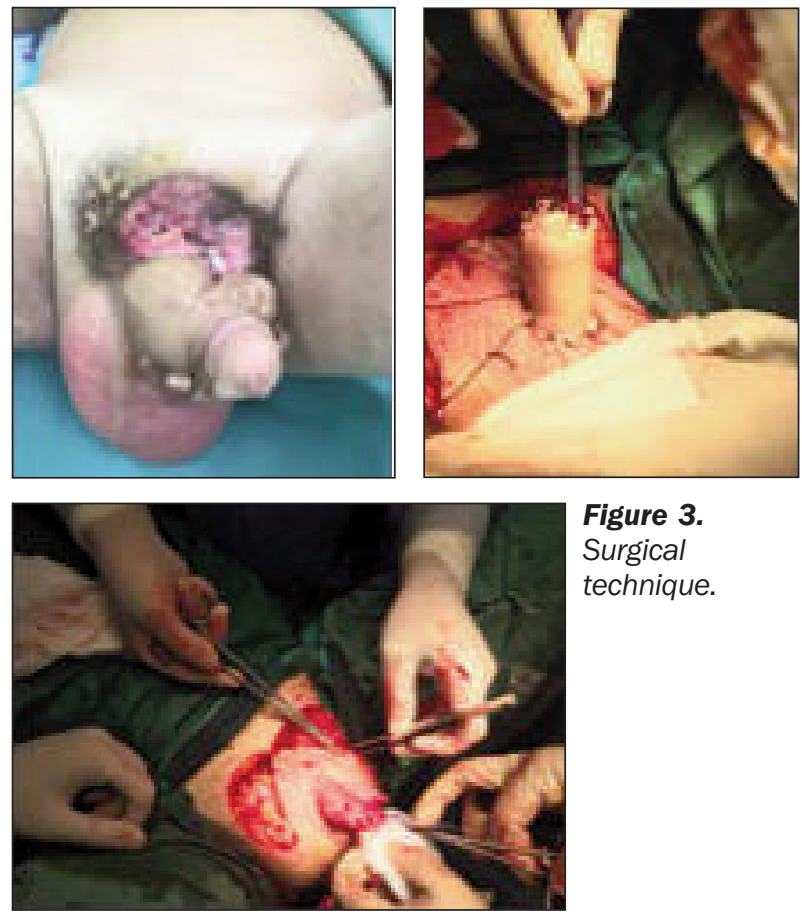

Figure 3.

Surgical

technique.

throstomy. The patient's penis was reconstructed with partial thickness skin graft. A dermotome was employed to achieve a graft, which was placed for immediate reconstruction after the oncologic resection (Figure 2). A foley catheter was placed for 7 days. The nodules in pubis were resected with macroscopic clearance as well. The large skin defect that was created as a consequence of this resection forced us to translocate the trunk of the penis in a more convenient location that could be covered by the scrotum (Figure 3). According to the pathology report, in gross inspection a tumor of $7.5 \times 4 \mathrm{~cm}$ in major dimension was noted involving the foreskin and glans of penis. In addition, multiple satellite nodules were noted in pubis. Histologically, the tumor was corresponded to a squamous cell carcinoma, exhibiting a solid pattern and minimal keratin production. In a small relatively area, tumor showed "basaloid features" with a solid pattern and peripheral palisading of nuclei, while tumor cells had small size and dark nuclei. Chromogranin and synaptophysin immunohistochemical stains were negative, so there was no neuroendocrine differentiation (Figures 4,5 ). The patient's post-operative healing was

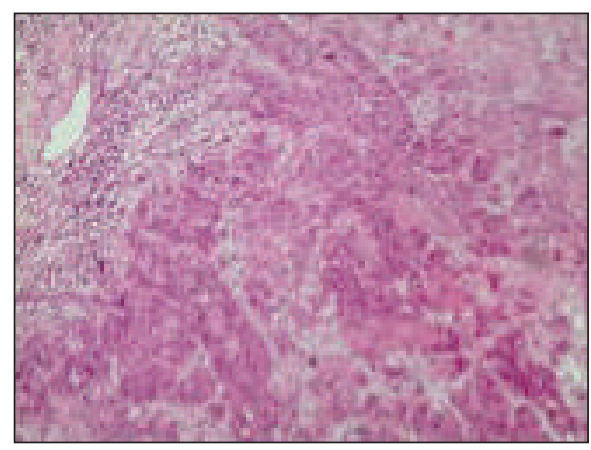

Figure 4.

Invasive squamous cell carcinoma (H\&EX100).

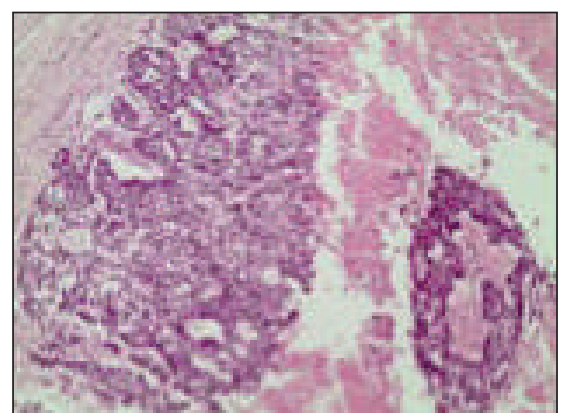

Figure 5.

Poorly differentiated squamous cell carcinoma with some "basaloid" features and hemorrhage (H\&EX100).

uneventful, and he was discharged after nine days. His HADS and GDS scores were already improved before discharge and a neuropsychologic reevaluation was scheduled six months later. Reevaluation scores in both questionnaires were found within the normal limits, while his subjective memory complaints were totally resolved. After 18 months of follow-up, our patient had not presented any voiding difficulty. Pelvic computed tomography and physical examination findings did not reveal any episode of recurrence or metastasis (Figure 6).

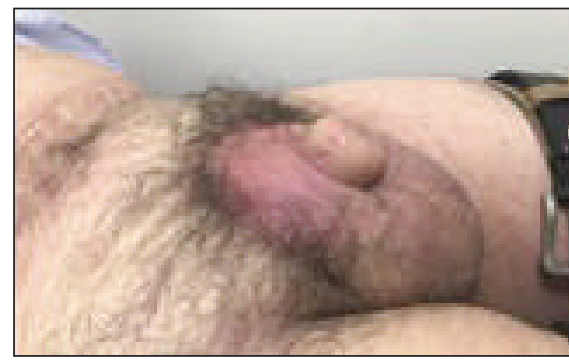

Figure 6.

18 months later.

\section{Discussion}

Penile amputation remains the standard therapy for patients with deeply invasive or high grade cancers. Partial penectomy should be considered in well selected patients exhibiting features for organ preservation strategies. Given the fact that the priority is always the cancer treatment, it seems possible that by using established reconstructive surgical techniques, erectile and voiding function, as well as psychological wellbeing of the patients can be preserved $(4,5)$.

Furthermore, it has been already demonstrated that penile cancer sufferers can exhibit significant psychological dysfunction. In our patient, subjective memory complaints were additionally present. Subjective memory complaints are associated either with sub-syndromal depression and anxiety in healthy cognitively normal adults or with mild cognitive decline (7). Therefore, there is a need to identify and assess adequate tools to measure neuropsychological comorbidity in this group of patients (4).

\section{Conclusions}

The treatment of the penile cancer has changed dramatically over the recent years. Organ-sparing treatments should be the first choice in penile squamous cell carcinoma, whenever this technique is adequately safe and efficient in oncological terms. Choosing the right tech- 
nique for the right patient is of paramount importance. Surgeon's experience accompanied with careful selection of patients suffering from this devastating disease but suitable for the safe organ-sparing surgical procedure, allows patients' access to preserving quality of life treatment options.

\section{Complance With ethical STANDARDS}

Conflict of interest: Authors declare that they have no conflict of interest.

Informed consent: Written informed consent was obtained from the patient for the publication of this Case Repost/any accompanying images.

\section{REFERENCES}

1. Vatanasapt V, Martin N, Sriplung H, Chindavijak K, et al. Cancer incidence in Thailand, 1988-1991. Cancer Epidemiol Biomarkers Prev. 1995; 4:475-83. PubMed PMID: 7549802.

2. Lynch HT, Krush AJ. Delay factors in detection of cancer of the penis. Nebr State Med J. 1969; 54:360-7. PubMed PMID: 4238912.
3: Horenblas S, Van Tinteren H, Delemarre JF, et al. Squamous cell carcinoma of the penis: accuracy of tumor, nodes and metastasis classification system, and role of lymphangiography, computerized tomography scan and fine needle aspiration cytology. J Urol. 1991; 146:1279-83. PubMed PMID: 1942279.

4. Maddineni SB, Lau MM, Sangar VK. Identifying the needs of penile cancer sufferers: a systematic review of the quality of life, psychosexual and psychosocial literature in penile cancer. BMC Urol. 2009; 9:8. doi: 10.1186/1471-2490-9-8.

5. Rybak J, Larsen S, Yu M, Levine LA. Single center outcomes after reconstructive surgical correction of adult acquired buried penis: measurements of erectile function, depression, and quality of life. J Sex Med. 2014; 11:1086-1091. doi: 10.1111/jsm.12417.

6. Hartung TJ, Friedrich M, Johansen C, et al. The Hospital Anxiety and Depression Scale (HADS) and the 9-Item Patient Health Questionnaire (PHQ-9) as Screening Instruments for Depression in Patients With Cancer. Cancer. 2017; 123:4236-4243. doi: 10.1002/cncr.30846.

7. Balash Y, Mordechovich M, Shabtai H, et al. Subjective memory complaints in elders: Depression, anxiety, or cognitive decline? Acta Neurol Scand. 2013; 127:344-50. doi: 10.1111/ane.12038.

\section{Correspondence}

Moulavasilis Napoleon, MD

napomoul@hotmail.com

Katafigiotis Ioannis, MD

Anastasiou Ioannis, MD

$1^{\text {st }}$ Department of Urology, National and Kapodistrian University of Athens, Laiko Hospital - Agiou Thoma str, Athens, 11527 (Greece)

Yiannopoulou Konstantina, MD

Department of Neurology, Henry Dunant Hospital Center, Athens (Greece)

Frangoulis Marios, MD

Plastic Surgeon, Academic Fellowship, National and Kapodistrian University of Athens, Laiko Hospital, Athens (Greece)

Liapis Georgios, MD

Department of Pathology, Medical School of Athens, National and Kapodistrian University of Athens (Greece)

Anastasiou Aikaterini, MD

Medical School of Athens, National and Kapodistrian University of Athens (Greece) 